\title{
Radiofrecuencia pulsada para el tratamiento del dolor crónico postherniorrafia inguinal. Reporte de caso
}

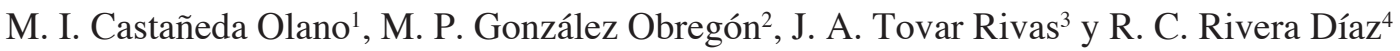

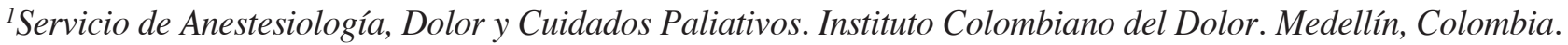 \\ ${ }^{2}$ Servicio de Anestesiología, Dolor y Cuidados Paliativos. Universidad CES. Instituto Colombiano del \\ Dolor. Medellín, Colombia. ${ }^{3}$ Servicio de Anestesiología - Anestesia Cardiovascular. Clínica Medellín, Nueva \\ Clínica Sagrado Corazón. Medellín, Colombia. ${ }^{4}$ Servicio de Anestesiología, Dolor y Cuidados Paliativos. \\ Departametno de Anestesia y Dolor. Universidad CES. Instituto Colombiano del Dolor. Medellín, Colombia
}

Castañeda Olano MI, González Obregón MP, Tovar Rivas JA, Rivera Díaz RC. Radiofrecuencia pulsada para el tratamiento del dolor crónico postherniorrafia inguinal. Reporte de caso. Rev Soc Esp Dolor 2014; 21(3): 182-184.

\begin{abstract}
Introduction: Postherniorrhaphy groin chronic pain is a clinical condition characterized by neuropathic pain mainly, disabling, with difficult pharmacological control, which has resulted in the search for different treatment tools.

Objective: To present the case of a patient diagnosed with postherniorrhaphy groin chronic pain, resistant to medical therapy, to whom was made a pulsed radiofrequency by means of which achieved pain control and their reintegration into working life.

Methods: A search was conducted on PubMed/Medline database, between the years 2000-2013.

Results: 25 papers were reviewed to describe the case.

Conclusion: In patients with postherniorrhaphy groin chronic pain resistant to pharmacological treatment, pulsed radiofrequency applied to the ilioinguinal, iliohypogastric and genitofemoral nerves is an effective alternative, available and safe with a low incidence of adverse effects or complications.
\end{abstract}

Key words: Inguinal hernia. Chronic pain. Pulsed radiofrequency. Nerve block. Ultrasonography.

\section{RESUMEN}

Introducción: El dolor crónico postherniorrafia inguinal es una condición clínica caracterizada por dolor frecuentemente neuropático, incapacitante, de difícil control farmacológico, lo cual ha derivado en la búsqueda de diferentes herramientas de tratamiento.

Objetivo: Presentamos el caso de un paciente con diagnóstico de dolor crónico postherniorrafia inguinal, refractario al tratamiento farmacológico, a quien se le realizó una radiofrecuencia pulsada con la cual se logró control del dolor y su reintegro a la vida laboral.

Métodos: Se realizó una búsqueda en la base de datos PubMed/Medline, entre los años 2000 y 2013.

Resultados: Se revisaron 25 artículos para la descripción del caso.

Conclusión: En pacientes con dolor crónico postherniorrafia inguinal, refractarios al tratamiento farmacológico, la radiofrecuencia pulsada aplicada sobre los nervios ilioinguinal, iliohipogástrico y genitofemoral, es una alternativa útil, disponible en nuestro medio y segura con una baja incidencia de efectos adversos o complicaciones.

Palabras clave: Hernia inguinal. Dolor crónico. Radiofrecuencia pulsada. Bloqueo de nervio. Ecografía.

\section{INTRODUCCIÓN}

El dolor crónico postherniorrafia inguinal (DCPHI) es una condición clínica caracterizada por dolor de predominio neuropático en la ingle, muslo y genitales, incapacitante y de difícil control farmacológico. Tiene una incidencia del 23 al $63 \%(1-4)$ y genera alteración funcional y/o sexual en un 2 al $10 \%$ de los casos (5-8). Sólo el $1 \%$ llega a la consulta especializada de dolor (9), algunos con poca respuesta al tratamiento convencional, siendo este grupo donde la radiofrecuencia pulsada (RFP) podría convertirse en una alternativa útil de manejo. 


\section{MATERIALES Y MÉTODOS}

Se realizó una búsqueda en la base de datos PubMed/ Medline, incluyendo revisiones sistemáticas, narrativas, ensayos clínicos, estudios retrospectivos, consensos de expertos y series de casos entre los años 2000 y 2013. Los términos de búsqueda $[\mathrm{MeSH}]$ fueron: "inguinal hernia", "chronic pain", "pulsed radiofrequency" "nerve block" "ultrasonography". Se evaluaron sólo artículos en humanos, adultos y publicados en inglés o español.

\section{DESCRIPCIÓN DEL CASO}

Paciente de 42 años remitido al Instituto Colombiano del Dolor (INCODOL) con 11 meses de dolor neuropático calificado en la escala visual análoga (EVA) 10/10 y alodinia en la ingle izquierda, posterior a una herniorrafia inguinal ipsilateral, irradiación al testículo y muslo, manejado con acetaminofén. Se inicia tratamiento farmacológico escalonado y multimodal con antiepilépticos, opioides, parches de lidocaína, antidepresivos tricíclicos y duales, en dosis y esquemas adecuados, sin mejoría de al menos un $50 \%$ a los dos meses de seguimiento, decidiéndose realizar un bloqueo guiado por ecografía de los nervios ilioinguinal, iliohipogástrico y genitofemoral izquierdos con anestésico local asociado a esteroide. Cuatro meses después califica el dolor $8 / 10$, con agudización al movimiento, hipersensibilidad en región inguinoescrotal izquierda, reflejo cremastérico positivo, percusión sobre espina iliaca anterosuperior negativa, practicándose un nuevo bloqueo, con el cual el paciente refiere una mejoría mayor al $50 \%$. Ante esta evolución se decide realizar una radiofrecuencia pulsada con cánula con punta activa de $10 \mathrm{~mm}$ en los nervio ilioinguinal, iliohipogástrico y genitofemoral izquierdos, previa prueba sensitiva a $0,2 \mathrm{mV}$ positiva, con los siguientes parámetros: $2 \mathrm{~Hz}$, fase activa de 20 milisegundos, control de temperatura $<42^{\circ} \mathrm{C}$, durante 10 minutos, aplicándose además anestésico local y esteroide en cada nervio. A la semana califica el dolor 2/10; al mes es calificado con 1/10, sin efectos adversos, reintegrándose a su actividad laboral y familiar. Mejoría sostenida en los controles a los 6 y 9 meses (EVA 2-3/10).

\section{RESULTADOS}

Al buscar en conjunto las palabras clave no se obtuvo ningún resultado, en forma combinada se obtuvieron 421 artículos y de manera independiente 118.542 artículos, teniéndose en cuenta 25 para esta revisión, descartándose los demás por título, resumen o contenido sin relación con el tema de revisión; cirugía, dolor y/o tratamiento en otras regiones anatómicas; relevancia, no disponibilidad o idiomas diferentes a inglés o español.

\section{DISCUSIÓN}

Descartadas otras causas de dolor inguinal como infección, hematomas, patologías urológicas, ginecológicas o ganancia secundaria por parte del paciente y con el antecedente quirúrgico se hace el diagnóstico de DCPHI (8-10). Para el tratamiento existen opciones farmacológicas como acetaminofén, antiinflamatorios no esteroideos, opioides, antidepresivos tricíclicos, gabapentinoides, antiepilépticos con respuestas variables en cada paciente (11-13); quirúrgicas como la neurectomía triple, con curación completa del 68 al $80 \%$ de los casos (14-16); intervencionistas como bloqueos nerviosos con anestésicos locales asociados a esteroides, con mejoría por cortos periodos y con los efectos adversos conocidos por su uso repetitivo (11-16). La neurolisis por radiofrecuencia (NRF) que mediante la aplicación de electricidad destruye el nervio y bloquea la transmisión del dolor, con buen control del mismo pero con una mayor duración del efecto (control dolor $77 \%$ y efecto 12,5 meses) cuando se compara con los bloqueos (control dolor 87,5\% vs. efecto 1,6 meses) $(17,18)$, aunque con la probabilidad de formación de neuromas y empeoramiento del dolor $(8,13)$. Existe un reporte de 10 casos, con crioablación a $-70{ }^{\circ} \mathrm{C}$ durante 3 minutos de los nervios ilioinguinal y genitofemoral expuestos quirúrgicamente, con mejoría del dolor de 77,5\% y disminución del uso de analgésicos en $80 \%$, teniendo solo una infección en una herida quirúrgica como complicación (19). Existe otro reporte de dos casos, con utilización de estimulación en médula espinal (EME) a nivel de T7 y T8, reportando a los 6 meses la suspensión del uso de opioides fuertes y manejo sólo con tramadol $50 \mathrm{mg}$ orales cada 6 a 8 horas a necesidad en un paciente y en el otro disminución de consumo a sólo metadona a dosis de $120 \mathrm{mg} /$ día (dosis previas de $240 \mathrm{mg} /$ día e hidrocodona $10 \mathrm{mg}$ cada 4 a 6 horas a necesidad), limitando el uso de EME para casos de respuesta fallida a todos los otros tratamientos (20).

La radiofrecuencia pulsada (RFP) es una técnica cuyo mecanismo de acción exacto no está del todo claro. Actualmente se investigan los cambios que a nivel molecular produce en la expresión de factores de inflamación y de proteínas, y también el efecto de los campos electromagnéticos sobre la neurona y en la transmisión del impulso doloroso $(21,22)$. En la última década ha ganado popularidad ya que, aunque libera una alta intensidad de corriente, lo hace en pulsos regulados alternantes que permiten la disipación del calor y con temperaturas que varían entre 38 y $42^{\circ} \mathrm{C}$; donde el daño tisular generado no alcanza a producir reacciones neuríticas que predispongan a la formación de neuromas, descritos por encima de $\operatorname{los} 45^{\circ} \mathrm{C}(21-25)$.

La técnica incluye la realización de pulsos de 20 milisegundos de duración a una frecuencia de $2 \mathrm{~Hz}$ y duración entre 4 y 12 minutos $(13,23-25)$. Se puede realizar a nivel de las raíces nerviosas de T12 a L2 $(13,23)$ con mejorías 
del 75 al $100 \%$ y con duración del efecto de 6 a 9 meses o directamente sobre los nervios afectados $(24,25)$ con mejoría del $70 \%$ a 3 meses de seguimiento. La ubicación de las estructuras nerviosas ya sea por guía fluoroscópica, ecográfica o tomográfica, hacen más certero el acceso al objetivo y por lo tanto brindan mayor seguridad al momento de la realización de la RFP (13,23-25). Controla la intensidad del dolor hasta en un $90 \%$ y en el 63 al $100 \%$ de los pacientes, por un periodo de 3 a 9 meses de seguimiento, sin efectos adversos o complicaciones reportadas (25), aunque la evidencia es débil a moderada en la revisión sistemática de Werner y su grupo (25), ellos manifiestan que la ausencia de evidencia de eficacia no significa evidencia de ausencia de eficacia, quedando como una buena terapia, con resultados promisorios, sin efectos adversos, costo efectiva y que requiere estudios con una mayor población y mejores diseños metodológicos para determinar su beneficio e impacto real en la salud y la calidad de vida de los pacientes (25).

\section{CONCLUSIÓN}

En pacientes con DCPHI, refractario al tratamiento farmacológico, la RFP sobre los nervios ilioinguinal, iliohipogástrico y genitofemoral, es una alternativa útil, disponible en nuestro medio, segura y con baja incidencia de efectos adversos o complicaciones.

\section{CORRESPONDENCIA}

María Isabel Castañeda Olano

Anestesiología, Dolor y Cuidados Paliativos

Instituto Colombiano del Dolor

Medellín, Colombia

e-mail: maisacast@gmail.com

\section{BIBLIOGRAFÍA}

1. Poobalan AS, Bruce J, Smith WC, King PM, Krukowski $\mathrm{ZH}$. A review of chronic pain after inguinal herniorrhaphy. Clin J Pain 2003;19(1):48-54.

2. Massaron S, Bona S, Fumagalli U, Battafarano F, Elmore U. Analysis of post-surgical pain after inguinal hernia repair: A prospective study of 1,440 operations. Hernia 2007;11 (6):517-25.

3. Nienhuijs S, Staal E, Strobbe L, Rosman C, Groenewoud H. Chronic pain after mesh repair of inguinal hernia: A systematic review. Am J Surg 2007;194(3):394-400.

4. Aasvang E, Kehlet H. Chronic postoperative pain: The case of inguinal herniorrhaphy. Br J Anaesth 2005;95(1):69-76.

5. Mikkelsen T, Werner MU, Lassen B, Kehlet H. Pain and sensory dysfunction 6 to 12 months after inguinal herniotomy. Anesth Analg 2004;99(1):146-51.
6. Dennis R, O'Riordan D. Risk factors for chronic pain after inguinal hernia repair. Ann R Coll Surg Engl 2007;89 (3):218-20.

7. O'Dwyer PJ, Alani A, McConnachie A. Groin hernia repair: Postherniorrhaphy pain. World J Surg 2005;29(8):1062-65.

8. Kehlet H. Chronic pain after groin hernia repair. Br J Surg 2008;95(2):135-6.

9. Loos MJ, Roumen RM, Scheltinga MR. Classifying postherniorrhaphy pain syndromes following elective inguinal hernia repair. World J Surg 2007;31(9):1760-5.

10. Ferzli G, Edwards E, Al-Khoury G, Hardin R. Postherniorrhaphy groin pain and how to avoid it. Surg Clin N Am 2008;88(1):203-16.

11. Ferzli GS, Edwards ED, Khoury GE. Chronic pain after inguinal herniorrhaphy. J Am Coll Surg 2007;205(2):33341.

12. Alfieri S, Amid PK, Campanelli G, Izard G, Kehlet H. International guidelines for prevention and management of postoperative chronic pain following inguinal hernia surgery. Hernia 2011;15(3):239-49.

13. Rozen D, Ahn J. Pulsed radiofrequency for the treatment of ilioinguinal neuralgia after inguinal herniorrhaphy. Mt Sinai J Med 2006;73(4):716-8.

14. Amid PK. Causes, prevention, and surgical treatment of postherniorrhaphy neuropathic inguinodynia: Triple neurectomy with proximal end implantation. Hernia 2004;8 (4):343-9.

15. Aasvang E, Kehlet H. Surgical management of chronic pain after inguinal hernia repair. Br J Surg 2005;92(7):795-01.

16. Zacest A, Magill S, Anderson V, Burchiel K. Long-term outcome following ilioinguinal neurectomy for chronic pain. Clinical article. J Neurosurg 2010;112(4):784-9.

17. Kastler A, Aubry S, Barbier-Brion B, Jehl J, Kastler B. Radiofrequency neurolysis in the management of inguinal neuralgia. preliminary study. Radiology 2012;262(2):7017.

18. Kastler A, Aubry S, Piccand V, Hadjidekov G, Tiberghien F. Radiofrequency neurolysis versus local nerve infiltration in 42 patients with refractory chronic inguinal neuralgia. Pain Physician 2012;15(3):237-44.

19. Fanelli R, DiSiena M, Lui F, Gersin K. Cryoanalgesic ablation for the treatment of chronic postherniorrhaphy neuropathic pain. Surg Endosc 2003;17(2):196-200.

20. Elias M. Spinal cord stimulation for post-herniorrhaphy pain. Neuromodulation 2000;3(3)155-7.

21. Chua NH, Vissers KC, Sluijter ME. Pulsed radiofrequency treatment in interventional pain management: Mechanisms and potential indications. A review. Acta Neurochir (Wien) 2011;153(4):763-7.

22. Bogduk N. Pulsed radiofrequency. Pain Med 2006;7(5):396407.

23. Rozen D, Parvez U. Pulsed radiofrequency of lumbar nerve roots for treatment of chronic inguinal herniorraphy pain. Pain Physician 2006;9(2):153-6.

24. Mitra R, Zeighami A, Mackey S. Pulsed radiofrequency for the treatment of chronic ilioinguinal neuropathy. Hernia 2007;11(4):369-71.

25. Werner M, Bischoff J, Rathmell J, Kehlet H. Pulsed radiofrequency in the treatment of persistent pain after inguinal herniotomy: A systematic review. Reg Anesth Pain Med 2012;37(3):340-3. 